\title{
STOP5: a hot debrief model for resuscitation cases in the emergency department
}

\author{
Craig Andrew Walker ${ }^{1,2}$, Laura McGregor ${ }^{3}$, Cameron Taylor ${ }^{1}$, \\ Sara Robinson ${ }^{1}$ \\ ${ }^{1}$ Emergency Department, Royal Infirmary of Edinburgh, Edinburgh, United Kingdom \\ ${ }^{2}$ Critical Care Department, St John's Hospital, Livingston, United Kingdom \\ ${ }^{3}$ Scottish Centre for Simulation and Clinical Human Factors, Forth Valley Royal Hospital, Forth Valley, \\ United Kingdom
}

Objective Team-based resuscitation in emergency departments (EDs) is an excellent opportunity for hot debriefs (HDBs). In creating a bespoke HDB model for emergency medicine resuscitations, we sought to optimize learning from clinical experience, identify team strengths, challenges, encourage honest reflection and focus on ways of improving future performance.

Methods Multidisciplinary ED focus groups reviewed existing models, identified benefits/barriers and created new frame works, testing and adapting further using fottage of a simulated complex resuscitation case. The new HDB tool was coined: "STOP5" (STOP for 5 minutes). Cases targeted were prehospital retrievals, major trauma, cardiac arrests, deaths in resuscitation, and staff-triggered. The framework details included a specifically scripted introduction followed by core elements that were $\mathrm{S}$ : summarize the case; $\mathrm{T}$ : things that went well; 0: opportunities to improve; P: points to action and responsibilities. Staffs were surveyed at 1 month prior then 6 and 18 months post-introduction. Data collection forms were used to identify and track hard outcomes/system improvements resulting directly from HDBs.

Results Potential benefits identified by respondents included: improved staff morale; team cohesion; improved care for future patients; promoting a culture for learning, patient safety and quality improvement. Ten process and equipment changes resulted directly from STOP5 over 12 months.

Conclusion We anticipate the STOP5 framework to be globally generalizable and effective for many ED teams.

Keywords Emergencies; Resuscitation; Clinical competence; Feedback; Education
elSSN: 2383-4625

Received: 25 September 2019

Revised: 8 November 2019

Accepted: 8 November 2019

Correspondence to:

Craig Andrew Walker

Emergency Department, Royal

Infirmary of Edinburgh, 51 Little France

Crescent, Edinburgh EH16 4SA, United

Kingdom

E-mail: craigawalker@nhs.net

ORCID

https://orcid.org/0000-0002-1946-0686

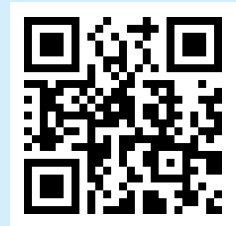

How to cite this article:

Walker CA, McGregor L, Taylor C, Robinson S. STOP5: a hot debrief model for resuscitation cases in the emergency department. Clin Exp Emerg Med 2020;7(4):259-266. https://doi. org/10.15441/ceem.19.086

This is an Open Access article distributed under the terms of the Creative Commons Attribution Non-Commercial License (https:// creativecommons.org/licenses/by-nc/4.0/). 


Wapsule
$\begin{aligned} & \text { What is already known } \\ & \text { Cummary } \\ & \text { Chical debriefing can improve performance and appears to work equally well within teams as it does for individuals. } \\ & \text { There is of intuitive, rapid, accessible hot debrief models in the clinical literature. }\end{aligned}$
$\begin{aligned} & \text { What is new in the current study } \\ & \text { We have designed, implemented and analysed the performance of a new hot debrief framework for use following re- } \\ & \text { suscitation cases in the emergency department, entitled "STOP5" (STOP for } 5 \text { minutes). It has proven in multiple quanti- } \\ & \text { tative and qualitative analyses to be effective. }\end{aligned}$

\section{INTRODUCTION}

The increasing number and variety of patient presentations to the emergency department (ED) produces an excellent environment for training, development and innovation. Evolving clinical diversity brings with it continuous opportunity to demonstrate improvement in patient care as the demands on ED services grow and interventions performed in the ED become increasingly complex. ${ }^{1,2}$ Across the world, emergency medicine (EM) teams face numerous common challenges and inevitably these will increase as multidisciplinary ED teams grow in size to meet rising demands. ${ }^{3,4}$ Importantly, according to a recent meta-analysis, teams that engage in well-conducted debriefs using interactive communication to learn and adapt together have been shown to outperform teams that do not use such a format. ${ }^{5}$

Leading a safe and healthy ED team requires the delivery of timely and effective peer support after particularly difficult or distressing clinical cases. ${ }^{6}$ The term 'moral injury' has been used to describe the resultant mental health difficulties of war veterans and is increasingly applied to psychological trauma presenting in frontline emergency and health services staff.? In preventing this, there is emphasis on fostering a safe clinical environment for all staff and patients whilst simultaneously supporting the development and specific needs of individual team members. ${ }^{8}$ This may be achieved in part by encouraging regular reflective analysis of patient care episodes, enabling the improvement of ED systems and the integration of professional cultures across the hospital. ${ }^{9}$

Human factors can be defined as "the organizational, individual, environmental and job characteristics that influence behavior in ways that can impact patient safety." ${ }^{10}$ In particular, organizational structure is known to impact on the interactions between different professionals within the ED team. ${ }^{9}$ It is recognized that analysis of process and individual team roles can be used to guide systems improvement, resulting in more consistent practice and decreasing the likelihood of future errors. ${ }^{11,12}$ Team debriefing is a consistently positive theme that features highly in systems improvement, team interactions and patient safety literature. .6,8,9 $^{-1}$

The 2013 white paper "Safety II" recommends a change in emphasis, suggesting we should examine more closely the many cases where thing go right and try to truly understand why, rather than focussing on the few cases when things go wrong. ${ }^{13}$ Accordingly, the greatest gains in ED systems improvement may be determined by examining the routine daily work of an ED team, rather than studying extreme or exceptional events.

As it is already established that debriefing can improve performance and appears to work equally well within teams as it does for individuals, ${ }^{5}$ we postulated that the simplest mechanism of introducing a model of clinical team debriefing was to begin in the ED resuscitation area. A hot debrief (HDB) is an interactive, structured team dialogue that takes place either immediately or very shortly after a clinical case. ${ }^{14}$ There is a surprising paucity of HDB models available that are suitable for use following ED resuscitation cases. To our knowledge, only one qualitative debriefing tool had been published for the ED population when we embarked on this project and that was created specifically for emergency pediatric intubation, cardiopulmonary resuscitation and defibrillation (i.e., a different setting and relatively limited case $\mathrm{mix})^{15}$

The aim of this study was to design, test and develop an HDB tool that was quick and easy for any staff member to use and that could be used to facilitate safer patient care, team development and quality improvement in the ED. Our primary outcome measure was staff satisfaction with the HDB tool. Secondary measures were the presence of hard outcomes and system improvements as a direct result of introducing the HDB model. 


\section{METHODS}

The Royal Infirmary of Edinburgh (RIE) is a large tertiary center with an ED seeing approximately 120,000 patients per year and a wide spectrum of illness and injury. In 2017, we established an HDB focus group consisting of seven EM consultants, three senior staff nurses, and four EM registrars along with four simulation leads representing the Scottish Centre for Simulation and Clinical Human Factors ${ }^{16}$ who acted as impartial facilitators.

Initially, the focus group worked together to determine the feasibility of developing a bespoke ED HDB tool by formally reviewing existing models from research literature used in other clinical contexts ${ }^{14,15,17,18}$ and identifying potential benefits and barriers to performing HDB in the ED. In smaller teams, the HDB focus group then created five individual original HDB frameworks in draft form. These five tools were evaluated in a timed simulated group HDB using predesigned footage of a complex simulated resuscitation case. Individuals volunteered to lead the group through an HDB to test and compare each tool, then to adapt further. Finally, themes were amalgamated and consolidated across the five models to produce the "STOP5" (STOP for 5 minutes) framework.

Our activities were judged to be a service evaluation project by our local quality improvement team and as such it was deemed that ethical approval was not required. There is existing ethical and Caldicott approval in place for the use of an audiovisual recording system for quality improvement and service evaluation

\section{STOP for 5 Minutes}

Thank the full team and ask "Is everyone ok?"

If YES then continue as below and STATE FIRST

-We are going to have a 5 minute team debrief

- Purpose is to improve quality of patient care; it is not a blaming session

- Your participation is welcomed but not compulsory

- All information discussed during this debrief is confidential

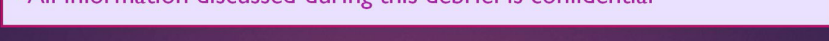

\section{Summarise the case}

Things that went well

Opportunities to improve

Points to action and responsibilities
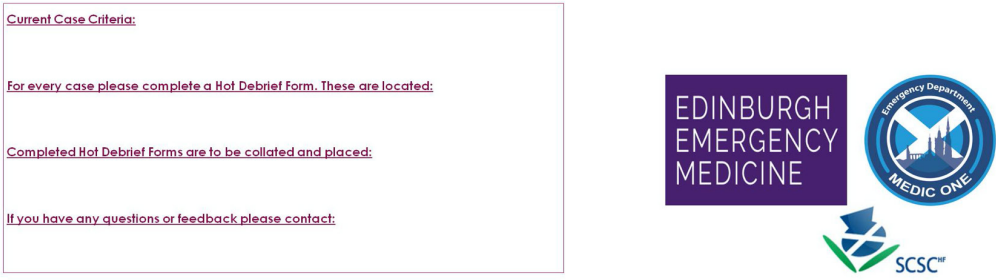

Fig. 1. STOP5 hot debrief framework. 
activities in our resuscitation areas. Additional informed consent from participants was therefore deemed to not be required.

\section{The STOP5 model}

The HDB framework created is entitled "STOP5" (Fig. 1). Any member of the resuscitation team may lead the HDB. As per the guidance poster, this individual thanks the full team and asks "ls everyone okay?" If yes, they continue and read out a series of statements: "We are going to have a 5-minute team debrief," "The purpose is to improve the quality of patient care; it is not a blaming session," "Your participation is welcomed but not compulsory," and "All information discussed during this debrief is confidential."

Thereafter, the team moves on to a group discussion, following the STOP5 framework: $\mathrm{S}$, summarize the case; $T$, things that went well; 0 , opportunities to improve; and $\mathrm{P}$, points to action and responsibilities.

The discussion phase is recorded on a separate STOP5 data collection form in order that points to be addressed may be acted upon (Fig. 2)..$^{19}$ The printed lower section of the HDB tool and the data collection form contain guidance on current case inclusion criteria, where to locate the data collection forms, what to do with completed forms and whom to contact regarding any questions or specific feedback.

Our initial inclusion criteria for STOP5 HDB case types were: major trauma, deaths in resus, and following a "prehospital callout." In our department, selected consultants and senior staff nurses form the "Medic One" service and attend prehospital callouts when requested by the Scottish Ambulance Service (e.g., en-

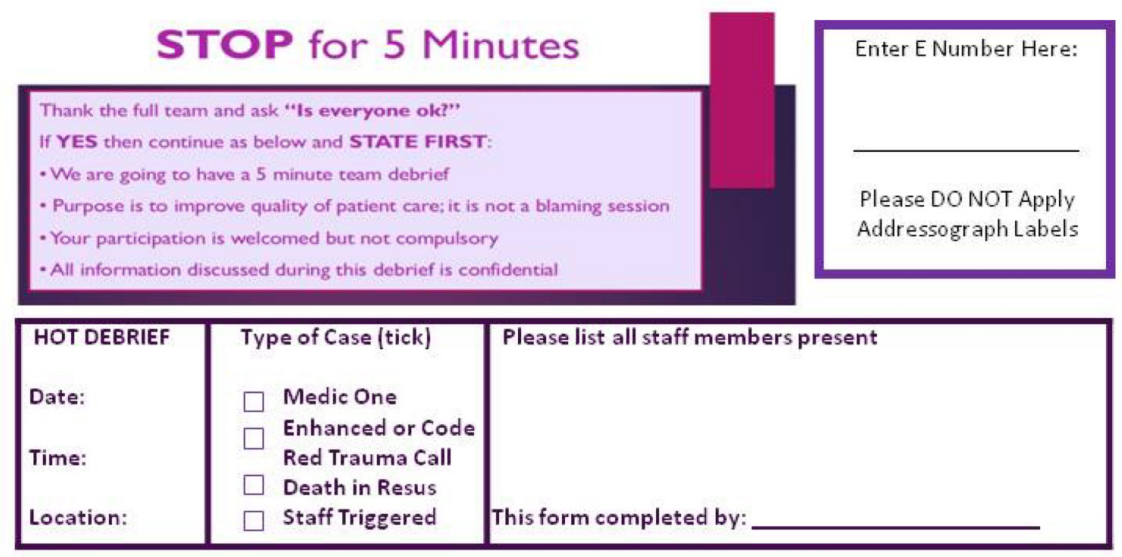

\section{Summarise the case}

\section{Things that went well}

\section{Opportunities to improve}

Points to action and $\begin{aligned} & \text { Include staff member to address } \\ & \text { each point }\end{aligned}$
responsibilities

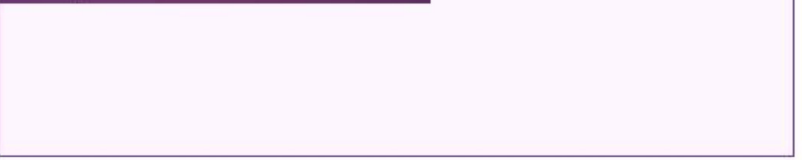

EDINBURGH EMERGENCY MEDICINE

\begin{tabular}{|l|l|}
\hline $\begin{array}{l}\square \text { HOT DEBRIEF not completed } \\
\text { Reason: }\end{array}$ & $\begin{array}{c}\text { Is a COLD DEBRIEF required? } \\
\text { YES } \square \quad \text { NO } \square\end{array}$ \\
\hline
\end{tabular}

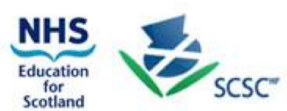

Fig. 2. Data collection form. 
trapments, difficult extrications, major road traffic collisions). We also included "upon request by any staff member" in our criteria. Hence, no potential resuscitation case type was formally excluded from the study.

\section{Quantitative and qualitative analyses}

One month prior to the introduction of the STOP5 HDB framework, ED staff working in RIE ED resuscitation areas were surveyed (through online and paper-based surveys) to ascertain how many had been involved in any form of HDB within the previous year and whether they felt that there should be more or less resuscitation team debriefs in the ED.

At 6 months post-STOP5 HDB introduction, online, and paperbased surveys were distributed to all ED staff working in resuscitation areas to obtain ratings for the HDB tool (using a Likert scale from 0 [very poor] to 4 [excellent]). Staff were surveyed regarding their confidence in leading, initiating and contributing to an HDB (scale from 0 [very unconfident] to 3 [very confident]) and perception of optimal HDB duration in minutes. Further to this, as RIE ED resuscitation rooms each have video and sound recording enabled for audit purposes, 15 sequential HDBs were retrieved for audio visual analysis and studied in detail regarding the specific content of the HDB, adherence to the STOP5 framework, choice of HDB leader, total duration in minutes and the representation of medical, nursing and prehospital staff.

At 18 months, ED staff were re-surveyed to ascertain STOP5 rating scores, the number of staff members who had been involved in HDBs in the last year, any perceived benefits or barriers to performance, and whether staff believed there should be more or less HDBs in the ED.

Separately, HDB data collection forms were prospectively analyzed to create a "resuscitation action log" in order to identify and track any hard outcomes and system improvements resulting directly from STOP5 HDBs. There had been no specific preexisting process in place through which to identify and track process improvements within resuscitation areas prior to the introduction of the STOP5 HDB tool.

\section{RESULTS}

At baseline, 19 of 40 staff surveyed had been involved in any form of HDB within the year preceding the creation of the STOP5 HDB tool. Medical staff (rather than nursing staff) led the debriefs in 24 of 26 cases (92.3\%). 39 out of 40 respondents (97.5\%) felt that there should be more HDBs in the ED.

The 6-month survey was completed by 30 staff members $(20$ doctors and 10 nurses). Twenty-seven (90\%) rated the usefulness

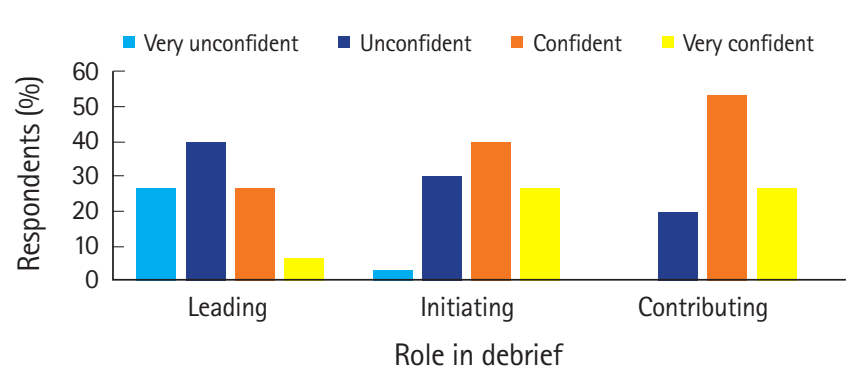

Fig. 3. Staff confidence levels in hot debrief roles.

of STOP5 HDBs as "good" to "excellent". The majority of respondents (22 of 30,73\%) felt that the optimal HDB duration was up to a maximum of 5 minutes. Staff confidence in leading, initiating and contributing to STOP5 HDBs is summarized in Fig. 3.

All 15 sequential HDBs retrieved for audio visual analysis adhered to the STOP5 HDB framework. Mean HDB length was 5.5 minutes (range, 1.5 to 13 minutes). Medical and nursing staff were represented in all debriefs; however, only 5 cases (33\%) involved paramedics or ambulance technicians. All 15 cases were led by a consultant or registrar and all demonstrated open discussion within the team.

In the 18-month review, there were 41 respondents (19 doctors, 19 nurses, and 3 clinical support workers). Seventy percent (29 of 41) had participated in any kind of HDB in the preceding year and 93\% of these (27 of 29) were in the STOP5 HDB format. All STOP5 debriefs were rated "good" to "excellent" by staff who had participated in them (32\% rated "excellent," 38\% "very good," and 30\% "good"), strongly suggesting that the STOP5 HDB remained highly valued by the team. Ninety-eight percent of respondents ( 40 of 41 ) believed that we should do even more HDBs in the ED.

The most frequent barriers encountered to performing HDBs were time pressures, departmental workload/busyness, competing clinical priorities (including a focus on the "4-hour" standard), and performance anxieties (Fig. 3), ensuring psychological safety and premature team dispersal during or at the end of resuscitation cases. Staff reported that waiting for other hospital specialty teams to return to the resuscitation room (as in enhanced/code red trauma cases when multiple specialties may have been involved) often resulted in the HDB being either significantly delayed and with fewer members of the ED team present, or no HDB being performed.

\section{Hard outcomes and system improvements resulting directly from STOP5 HDBs}

Over the past 12 months, there have been 10 "hard outcomes," defined as process and equipment changes as a direct result of a 
Table 1. Direct process and equipment changes following STOP5 HDB

\begin{tabular}{|c|c|c|c|}
\hline Issue no. & Issue identified during STOP5 HDB & Intervention performed & Recurrence of issue? \\
\hline 1 & Procedural error due to omission of step in preparation & $\begin{array}{l}\text { Checklist created and implemented } \\
\text { Added to weekly "Resus Friday" staff training sessions }\end{array}$ & No \\
\hline 3 & Resuscitation room phone not accepting incoming calls & $\begin{array}{l}\text { Reported and fully repaired within } 1 \text { week } \\
\text { Ongoing monitoring }\end{array}$ & No \\
\hline 5 & Faulty door in resuscitation room & Reported and fully repaired by maintenance staff within 3 days & No \\
\hline 6 & Emergency mobile phones unable to make outgoing calls & $\begin{array}{l}\text { Reported: identified service provider had accidentally cut off phones } \\
\text { Fixed same day } \\
\text { Ongoing monitoring }\end{array}$ & No \\
\hline 7 & Machine fault & $\begin{array}{l}\text { Reported } \\
\text { Fixed in } 1 \text { day }\end{array}$ & No \\
\hline 10 & Discrepancy between paper and online checklists & $\begin{array}{l}\text { Identified during HDB } \\
\text { Checklists updated same day }\end{array}$ & No \\
\hline
\end{tabular}

STOP5, summarize the case, things that went well, opportunities to improve, points to action and responsibilities for 5 minutes; HDB, hot debrief; ED, emergency department.

STOP5 HDB (Table 1). An additional 14 opportunities to improve were identified during HDB cases and highlighted as important information to share with the entire ED team during medical and nursing handover meetings.

\section{DISCUSSION}

Our aim was to design a model for HDB that was easy to use and would aid in the development of individual staff, all ED team members, and integral ED systems. To do this, the focus group recognized that the HDB format must provide a supportive environment and enable team members to speak freely should they identify critical performance or teamworking issues. During the initial HDB focus group discussions, it was acknowledged that resuscitation room cases can be emotionally challenging and may benefit from later formal cold debriefing with some team members requiring additional support thereafter.

Our STOP5 model for HDB begins with a series of set statements defined by the initial HDB focus group. These words are deliberately set out to provide a clear starting point and set the scene, enabling any individual team member to lead the HDB. This element of the tool aims to minimize potentially negative influences of perceived authority gradients: it was deemed essential by the multidisciplinary HDB focus group to help ensure a psychologically safe environment, where participation is valued (though voluntary) and individuals are supported to speak honestly and provide team-orientated feedback. After this preamble, a brief summary of events is provided. The team leader may be best-placed to do this, particularly if a 'hands-off' leadership style has been adopted with oversight of the entire case. It is accepted that team members may have become necessarily task-focused during elements of the resuscitation and this may have impacted upon their overall situational awareness.

The next two stages of the framework ('Things that went well' and 'Opportunities to improve') may appear similar to Pendleton's rules ${ }^{20}$ at first glance in that they focus on positive aspects before moving on to discuss what could be done better. In reality, the STOP5 approach differs in several important aspects. Most importantly, Pendleton's rules represent a predominantly paternalistic approach (with "learner" and "facilitator") and may imply that the facilitator knows the "correct answers." In the STOP5 model, any team member can lead the HDB and viewpoints of all members are valued equally. During the STOP5 HDB, there is no assumption that the team leader provides the solutions to issues that have arisen. Interestingly, this may be highlighted by the survey findings that cardiac arrest cases were voted to be both the category least and the category second-most likely to benefit from HDBs (with only major trauma scoring more highly as the category thought most likely to benefit from HDBs). We postulate that the diverse mix of staff members in ED resuscitation teams means that, whilst senior staff may not self-report a personal need for emotional support or new additional learning from leading or debriefing seemingly straightforward cases, more junior team members with less ED experience can in fact learn a great 
deal when given such opportunities. Consequently, what is important for one team member may be less so for another and senior ED staff may not be best-placed to judge this accurately. Our results suggest that we cannot presume to know or understand the emotional upset experienced by our ED teams unless we provide the forum to ask and the time to reflect. Perhaps making explicit the opportunity for emotional support and decompression is, in itself, fundamentally important in such circumstances, even if no practice-changing 'Opportunities to Improve' are identified.

The last step in the STOP5 model is "Points to action and responsibilities." Formalizing this step focuses the team and clearly allows process problems to be identified and solutions sought (Table 1). Output from STOP5 HDB records are shared with the wider ED and hospital teams through our safety newsletter, medical and nursing handovers and during separate education and training sessions.

The STOP5 HDB model has been welcomed by the entire multidisciplinary team and rating results suggest they believe it to be highly useful. The video data clearly demonstrates that the format is being adhered to and promotes open discussion and team reflection. We are confident that team members feel able to speak openly in this psychologically safe environment. Video analysis and staff surveys confirm that 5-minute HDBs are both desirable and feasible (mean HDB length 5.5 minutes). We feel that the brevity of the STOP5 framework is essential in achieving this timeframe: the set statements are short; the mnemonic is brief and easy to remember; there are no extraneous or repetitive steps. Nevertheless, STOP5 posters are clearly displayed in all of our resuscitation rooms to aid recall, help remind staff that they might want to perform an HDB and to help boost the confidence of those leading the sessions.

In all surveys, staff were keen to provide free comments as feedback. Common themes noted on the benefits of STOP5 HDBs were that individual staff members valued being given the opportunity to verbally thank each other for their hard work and to recognize that some resuscitation cases were particularly emotionally challenging. STOP5 HDBs were seen to highlight that despite being a large staff group, we prioritize team members' wellbeing and are supportive of one another. This was felt to improve staff morale and enhance overall team cohesion. Some staff described how STOP5 HDBs provided a valuable pause in workload during which to address misunderstandings and to resolve conflict. Debriefing soon after resuscitation cases ended was thought to be especially helpful through increasing factual recall, thereby increasing the likelihood of accurately identifying learning points, aiding staff development and improving patient care and safety.

Earlier studies have shown that postevent debrief improves team performance over time by approximately $20 \% .{ }^{5}$ Currently, we do not have a formal mechanism for measuring individual or team performance to show change related to our model. That team members feel there should be more HDB indicates that they feel there is inherent value in them.

This study has several limitations. It was performed at a single site and those team members represented within the initial HDB focus group were volunteers, the majority having an existing interest in teamworking and debriefing formats. There may be further bias in the respondents to the staff surveys, though this data was collected and collated anonymously and sent to all staff members working in our resuscitation rooms. Having video-recording readily available for audit purposes in the resuscitation rooms might be deemed as analyzing events vulnerable to the Hawthorne effect. ${ }^{21}$ However, local experience over 5 years of sustained observation and constant in-situ video and audio recording would contradict this assumption. Our staff are also aware that access to the recordings are controlled by strict information governance policies and they are analyzed for audit purposes only on a project-by-project basis. Another limitation may be the relatively low presence of prehospital-based personnel during the HDBs: video analysis demonstrated that they were present in only $1 / 3$ of cases. Whilst it is encouraging that our staff actively sought out the prehospital personnel when planning to start STOP5 HDBs, there will inevitably be situations when these individuals cannot be present. In future work, we intend to streamline processes for regular communication of outcomes from STOP5 HDBs to prehospital colleagues. Separately, there was no formal structure in place by which to identify and track hard outcome/process improvements to our resuscitation areas prior to the introduction of STOP5, and so it was not possible to identify whether STOP5 HDBs significantly affected the rate or number of such improvements.

In summary, the STOP5 HDB model is rapid, easy to use and effective in a busy ED setting. We anticipate the tool to be globally generalizable and effective for many ED teams. The next stage of development will involve extending and increasing use of this tool in both the ED and other clinical areas within our hospital. We would like to put systems in place to allow regular feedback to prehospital staff and other hospital specialties for situations in which they could not be present for the STOP5 HDB, and to do a more detailed evaluation on the role of the model in staff wellbeing.

\section{CONFLICT OF INTEREST}

No potential conflict of interest relevant to this article was reported. 


\section{ACKNOWLEDGMENTS}

We would like to thank Dr Robert Jack and Dr Cameron Taylor for conducting interim surveys under the supervision of Dr Craig Andrew Walker.

\section{REFERENCES}

1. UK Parliament. Health Committee: written evidence for the Royal College of Emergency Medicine (ES 07) [Internet]. London: UK Parliament; 2013 [cited 2019 Nov 8]. Available from: https://publications.parliament.uk/pa/cm201314/cmselect/ cmhealth/171/171we06.htm.

2. Morley C, Unwin M, Peterson GM, Stankovich J, Kinsman L. Emergency department crowding: a systematic review of causes, consequences and solutions. PLoS One 2018;13:e0203316.

3. Lowthian JA, Curtis AJ, Cameron PA, Stoelwinder JU, Cooke MW, McNeil JJ. Systematic review of trends in emergency department attendances: an Australian perspective. Emerg Med J 2011;28:373-7.

4. Anandaciva $S$, Thompson J. What is happening to waiting times in the NHS? [Internet]. London: The King's Fund; 2017 [cited 2019 Nov 8]. Available from: https://www.kingsfund. org.uk/publications/articles/nhs-waiting-times.

5. Tannenbaum SI, Cerasoli CP. Do team and individual debriefs enhance performance? A meta-analysis. Hum Factors 2013; 55:231-45.

6. Lateef F. Grace under pressure: leadership in emergency medicine. J Emerg Trauma Shock 2018;11:73-9.

7. Murray $E_{1}$ Krahe $C$, Goodsman D. Are medical students in prehospital care at risk of moral injury? Emerg Med J 2018;35: 590-4.

8. Weller J, Boyd M, Cumin D. Teams, tribes and patient safety: overcoming barriers to effective teamwork in healthcare. Postgrad Med J 2014;90:149-54.

9. Seow E. Leading and managing an emergency department: a personal view. J Acute Med 2013;3:61-6.

10. Ives $C_{1}$ Hillier $S$. Human factors in healthcare: common terms [Internet]. Clinical Human Factors Group; 2015 [cited 2019 Nov 8]. Available from: https://chfg.org/what-are-clinicalhuman-factors/.
11. National Quality Board. Human factors in healthcare: a concordat from the National Quality Board. Leeds: NHS; 2013 [cited 2019 Nov 8]. Available from: https://www.england.nhs. uk/wp-content/uploads/2013/11/nqb-hum-fact-concord.pdf.

12. Clinical Human Factors Group. "What good looks like" prompt cards: observed behaviours of organisations that apply human factors in their daily work [Internet]. Clinical Human Factors Group; 2013 [cited 2019 Nov 8]. Available from: https://chfg. org/what-good-looks-like/.

13. Hollnagel E, Wears RL, Braithwaite J. From Safety-I to SafetyII: a white paper [Internet]. Resilient Health Care Net; 2015 [cited 2019 Nov 8]. Available from: https://www.england.nhs. uk/signuptosafety/wp-content/uploads/sites/16/2015/10/ safety-1-safety-2-whte-papr.pdf.

14. Kessler DO, Cheng A, Mullan PC. Debriefing in the emergency department after clinical events: a practical guide. Ann Emerg Med 2015;65:690-8.

15. Mullan PC, Wuestner E, Kerr TD, Christopher DP, Patel B. Implementation of an in situ qualitative debriefing tool for resuscitations. Resuscitation 2013;84:946-51.

16. NHS Forth Valley. The Scottish Centre for Simulation and Clinical Human Factors [Internet]. Larbert: Forth Valley Royal Hospital; 2012 [cited 2019 Nov 8]. Available from: https://scschf. org.

17. Ahmed M, Arora S, Russ S, Darzi A, Vincent C, Sevdalis N. Operation debrief: a SHARP improvement in performance feedback in the operating room. Ann Surg 2013;258:958-63.

18. Sawyer T, Loren D, Halamek LP. Post-event debriefings during neonatal care: why are we not doing them, and how can we start? J Perinatol 2016;36:415-9.

19. Allen JA, Reiter-Palmon $R$, Crowe J, Scott C. Debriefs: teams learning from doing in context. Am Psychol 2018;73:504-16.

20. Pendleton D, Schofield T, Tate P, Havelock P. The consultation: an approach to learning and teaching. Oxford: Oxford University Press; 1984.

21. Oswald D, Sherratt F, Smith S. Handling the Hawthorne effect: the challenges surrounding a participant observer. Rev Soc Stud 2014;1:53-73. 